\title{
EUROBUILD Y EL FUTURO DE LA CONSTRUCCION
}

\author{
(EUROBUILD AND THE FUTURE OF CONSTRUCTION)
}

Fernando Aguirre de Iraola, Dr. Arquitecto

IETcc/CSIC

070-15

\section{RESUMEN}

Se da cuenta en este artículo del cambio de actividad y de nombre de la prestigiosa asociación europea EUROPREFAB, convertida hoy en EUROBUILD, como consecuencia de no existir ya un interés tan grande en las técnicas de la prefabricación como hubo en las décadas anteriores, y de presentarse un brillante futuro para la investigación de la construcción, en función de la forma que se prevé hayan de adoptar las industrias de este sector en el año 2000.

Se pasa una breve revista a los principales temas debatidos en la conferencia que tuvo lugar en Scheweningen (La Haya)

-organizada por esa Asociación-, temas que versaron sobre la influencia de la Electrónica en la industria de la construcción; la influencia de la coordinación dimensional en el diseño y en la ejecución de las obras; los métodos de trabajo y las nuevas relaciones entre los participantes en el proceso constructivo, los tipos y formas del habitat bajo la influencia de los medios audiovisuales $y$ de la telemática; $y$ nuevos materiales, métodos y procedimientos de construcción.
SUMMARY

In this article an account is given of the change in activity and name of the prestigious European association EUROPREFAB, which has now become EUROBUILD, as a result of the fact that there is no longer such great interest in prefabrication techniques as was the case in previous decades, and the dawning of a brilliant future for construction research, concerning the expected form to be adopted by the industries in this sector in the year 2000.

The main topics discussed at the conference held in Scheweningen (The Hague) - organised by that Association are briefly reviewed. These topics dealt with the influence of Electronics in the construction industry, the influence of dimensional co-ordination in building designs and their execution; work methods and the new relationship between participants in the construction process, the types and forms of habitat under the influence of audiovisual and telematic media; and new materials, methods and procedures of construction.
El asiduo lector de esta revista habrá seguido las actividades de la EUROPREFAB, prestigiosa Asociación internacional que integraba organismos de los principales países europeos, tanto en el ámbito de las empresas de construcción («Association of Building Component Manufacturers Limited» inglesa o la «Fédération des Travaux Publics» francesa), como en el área de la investigación («Studiengemeinschaft für Fertigbau» alemana o la «Epitéstudomanyi Egyesület»-sociedad científica para la investigación en la construcción - húngara).

España, a través de los dos socios de pleno derecho, el Instituto Eduardo Torroja y la Confederación Nacional de la Construcción, ha colaborado durante muchos años en las labores de la EUROPREFAB, y ha podido estar al día en la filosofía, las técnicas y en los sistemas relativos, especialmente, a la industrialización y a la prefabricación.

Sin embargo, como es de general conocimiento, en los últimos años se ha podido comprobar, no solamente en España, sino en toda Europa, una acusada recesión de los procedimientos industrializados, especialmente de la prefabricación pesada. $Y$, en consecuencia, en la reciente Asamblea General de la Asociación, celebrada en Belgrado, el Consejo ejecutivo aprobó una alteración de sus objetivos e incluso de su nombre.

En lugar de promocionar la prefabricación y otros métodos industrializados de construcción, como se ha hecho desde su fundación en 1965, se acordó que la asociación deberá investigar y promocionar nuevas técnicas y métodos de construcción y cambiar su nombre por el de EUROBUILD, con el fin de que pueda desprenderse de la imagen restrictiva que hasta ahora ha tenido y que deberá. mirar hacia el futuro y hacia la forma que haya de adoptar la industria de la construcción en el año 2000. Con todos los dramáticos cambios que están teniendo lugar en la aplicación de los microcomputadores tanto a las comunicaciones y a la robotización como a las nuevas técnicas de fabricación, la industria tal y como la hemos conocido, será seguramente sobrepasada por procedimientos más sofisticados, y es a la nueva organización con sus miembros, entre los cuales se incluyen la mayoría de los países europeos, a la 
40

Informes de la Construcción. Vol. 36, n. ${ }^{\circ} 363$, agosto-septiembre, 1984

que corresponde investigar e informar sobre las mejoras y las más avanzadas técnicas de cada país miembro, de manera que con ello se beneficie en conjunto la industria de la construcción europea.

Como primer paso importante que subraye el cambio de dirección emprendido, se ha celebrado en Holanda (cerca de La Haya), y organizado por el miembro holandés, el Progresbouw una Conferencia o coloquio internacional, con el título de la «construcción en el futuro», a la cual han asistido casi un centenar de técnicos y especialistas europeos en el tema.

Los principales temas debatidos fueron propuestos según las siguientes ponencias:

- Influencia de la electrónica en la industria de la construcción.

- Influencia de la coordinación dimensional.

- Perspectivas del futuro de la construcción.

- Métodos de trabajo y nuevas relaciones entre los participantes en el proceso constructivo.

- Tipos y formas del habitat bajo la influencia de los medios audiovisuales y de la electrónica.

- Nuevos métodos y procedimientos de construcción.

En su informe, el actual Presidente de la EUROBUILD y delegado del Reino Unido, W. Forgham, afirmaba que la revolución que está teniendo lugar debido a los avances de la electrónica y, particularmente, al micro-computador, es el hecho más importante acaecido en la industria de la construcción durante este siglo, y que está afectando ya y afectará en mucho mayor grado al individuo y a las colectividades, tanto en el hogar, como en la oficina, la empresa, la factoría o la obra.

Uno de los objetivos de su ponencia fue provocar la discusión de los aspectos de la comunicación y de la coordinación en la industria del futuro, teniendo en cuenta que la comunicación y un completo entendimiento entre todos los miembros del equipo de construcción, bien sea el cliente, el usuario, el arquitecto, el constructor o el promotor, es de la máxima importancia.

En el pasado, la comunicación ha sido dependiente en su mayor parte de la palabra hablada, pero esta forma de expresión es muy susceptible de falsedad en interpretación. La construcción emplea muchos conceptos y términos comunes, que al acceder a la interpretación internacional propia de Mercados comunes y del futuro deseable de intercambio de materiales, elementos y sistemas, originan diferencias que dificultan el proceso constructivo. Y la experiencia ha demostrado que la palabra hablada habrá de ser reforzada por algo más. Seguramente, el futuro aportará los medios para registrar la palabra seguida de traducción instantánea y almacenamiento en la memoria, lo cual nos permitirá recogerla cuando lo deseemos, y en cualquier lugar que nos hallemos. Esto nos permitirá no solamente clarificar nuestro pensamiento, sino también adoptar decisiones inmediatas y positivas.

Por otra parte, el mundo visual de la comunicación ahorrará la necesidad de viajes rutinarios e improductivos, ya que éstos podrán sustituirse por la comunicación basada en el video.

También la velocidad en la comunicación experimentará un incremento revolucionario, ya que los computadores constituirán el núcleo de cualquier sistema de comunicación del futuro, transformando el discurso hablado en impulsos eléctricos que podrán ser almacenados en las correspondientes memorias. Al recuperar esta información y al distribuirla a través de las más modernas redes de comunicación (cables de fibras ópticas, satélites, rayos láser), se podrá lograr una documentación prácticamente infinita para seleccionarla después de un modo inmediato.

$Y$ si se pretende que el proceso constructivo global se realice de un modo científico, para lo cual será necesaria una perfecta coordinación entre todos sus elementos integrantes, la informátic ${ }^{-}$ha de ser el gran adelanto técnico de los últimos años, imprescindible para el futuro de la construcción.

Otra ponencia interesante presentada en el coloquio por el Secretario General de la EUROBUILD y de la Fédération du Bâtiment francesa, $M$. Vandenkerckhove, y resultado de los estudios realizados por el IDATE (Institut pour le Développement et l'Aménagement des Télécommunications), flevaba por título «Tipos y formas de habitat bajo influencia de los medios audiovisuales y de la electrónica». Se extracta de ella las principales ideas:

El citado Instituto señala las posibles evoluciones de la vida humana en el siglo XXI, a base de los nuevos medios ya existentes (terminales telemáticos, cadenas audiovisuales, pantallas gigantes, ordenadores domésticos, etc.) y a partir de los sistemas electrónicos:

- la telemática, el control de abonados y su red para la vivienda;

- la peritelevisión, las cajas de interconexión de los sistemas audiovisuales;

- el micro-ordenador doméstico polivalente;

- la automación de regulación o de programación. 
Si la integración de los equipos electrónicos en el Habitat se halla, en la actualidad, influenciada en gran medida por la concepción tradicional de la distribución de espacios y por los valores simbólicos ligados a ellos, su multiplicación podría traducirse por una reestructuración de ciertos espacios y una redistribución de las superficies de la vivienda.

Imaginando la casa del futuro, los nuevos bienes electrónicos caracterizarán la evolución del teléfono al controlador de abonado y a la red de apartamento, que será una red múltiple encargada de dar servicio a la mayor parte de las habitaciones de la vivienda.

La conexión se realiza de momento con la red de integración telefónica y de datos (RITD) de cobre, que permitirá la unión con terminales tales como tele-alarma, tele-vigilancia, teléfono videotex, fac-simil. Pero esta red de cobre será próximamente reemplazada gracias al programa de transmisión por medio de fibras ópticas, programa que prevé instalar en Francia quince millones de hogares electrónicos en 1985. Las posibilidades que ofrece este sistema son considerables, y se podrán añadir a los terminales citados, aquéllos ligados a la recepción o a la emisión de señales video o de sondeo de alta fidelidad.

Otra evolución previsible es la determinada por la toma PERITEL normalizada, que ha favorecido la aparición de periféricos de televisión que permiten la ramificación de magnetoscopio, consola de juegos, videodisco, micro-ordenador, adaptadores videotext o de antenas de recepción directa. (Fig. 1).

Los constructores prevén en el futuro una organización de diferentes elementos de la cadena audio-visual, alrededor de un mando a distancia, denominada SIDAV (sistema de interconexión dinámica y audiovisual).

Un superordenador doméstico se encargará de realizar el conjunto de funciones ligadas a la

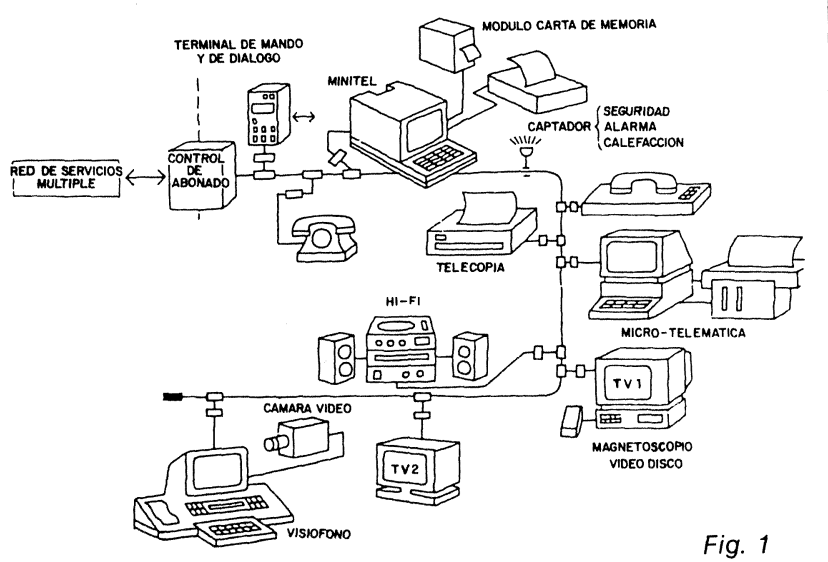

gestión del habitat y de las actividades de sus ocupantes.

Todos estos adelantos supondrán, naturalmente, unas importantes modificaciones en la distribución de las superficies de los espacios en los edificios futuros. Concretándonos a la vivienda, la transformación del cuarto de estar en un «hogar electrónico» facilitará un acceso colectivo a los equipos que allí se han de concentrar. $\mathrm{La}$ interpretación de los productos video y de la cadena de Hi-Fi, el desarrollo del videoproyector doméstico, y posteriormente de la pantalla de grandes dimensiones combinada con la penetración de la imagen de alta definición, deberán constituir las principales etapas para la creación de estos espacios, que la perspectiva necesaria para la contemplación del muro o pared-pantalla exigirá una distancia mínima de tres veces su altura.

Los estudios del IDATE se han extendido sobre las correlaciones posibles entre los tres modelos diferentes que configurarán la realidad socio-económica del mañana, en función de los nuevos equipos de bienes electrónicos, tanto domésticos como de oficina, talleres, centros de enseñanza, etc., etc., y que determinarán una morfología de los espacios habitables: mantenimiento y generalización del habitat; enriquecimiento de las funciones en la zona del habitat, y empobrecimiento de estas funciones.

¿Cuál será la importancia futura del trabajo a domicilio? El tele-trabajo, la tele-enseñanza, la tele-compra o venta, la tele-financiación, etc., modificarán fundamentalmente la vida actual, y podrán incluso producir una considerable reducción en el flujo del transporte, lo cual es sumamente interesante para el arquitecto urbanista.

La tercera Revolución industrial, que introduce de ahora en adelante nuevas aspiraciones en los pueblos, engendrará nuevas necesidades en el plano social y, por consiguiente, unas inversiones en el campo de los equipos y de las infraestructuras. Esta revolución será también la fuente de nuevos equipos industriales, de una redistribución de la actividad mundial y también de una remodelación de las infraestructuras y de los equipos, en particular, en el área de la vivienda.

Otra ponencia de interés fue la titulada «Métodos de trabajo y nuevas relaciones entre los participantes en el proceso de construcción».

En esta aportación, original de M.J. Le Gall, se destacaba el interés que puede ofrecer EUROBUILD para potenciar la comparación de situaciones y problemas específicos de cada país, y tratar de hallar soluciones para resolver la grave crisis por la cual atraviesa la industria de la construcción europea, basada principalmente en la inadecuación entre la solvencia y el costo global de la edificación. 
La sistemática propuesta en la ponencia para racionalizar el proceso encaminado a resolver el problema era:

- Conocer las exigencias y valorar, en función de ellas, total o parcialmente los componentes, utilizando las técnicas y útiles modernos que la Industria tradicional ha puesto a punto y ha experimentado.

- Definir las funciones que deben intervenir en la preparación de la respuesta a las necesidades, sin dejarse aprisionar por las actuales estructuras profesionales ni por sus formas de relacionarse habituales e incluso a veces ancestrales.

- Ordenar las tareas a realizar y precisar las funciones que las deban responsabilizar.

- Conocer los cortos resultados de inversión y utilización.

- Concebir, por último, los múltiples hilos estructurados capaces de intervenir en este cuadro.

Por último, y antes de cerrar esta referencia, el autor del presente artículo desearía dar cuenta de su intervención dentro de uno de los tres grupos de trabajo que se organizaron como final del coloquio internacional, con objeto de debatir los temas por separado. Haciendo referencia a las conversaciones mantenidas hace años con el desaparecido y célebre arquitecto Richard Neutra, opinó que no se podría obtener una panorámica completa sobre el habitat y la construcción del futuro sin un estudio científico de las exigencias funcionales del hombre del mañana. Citó las colaboraciones de Neutra con psicólogos, biólogos, sociólogos, etc., que le hicieron concebir las ciudades del siglo venidero de una manera totalmente distinta a la actual.

Recordó también los trabajos del Dr. checo Jokl, presentados al CIB en el Congreso de Edimburgo («Behaviour and requeriments of users inside the dwelling (fonctional analysis) and implications for design of dwelling units»).

El Dr. Jokl, al estudiar las interacciones para el desarrollo de la investigación a nivel de proyecto, tomando como base la identificación de las agregaciones de funciones compatibles con su correspondiente traducción en términos espaciales, dimensionales y de organización, establecía los siguientes puntos:

- Análisis de la agregabilidad y de la incompatibilidad de las funciones.

- Individualización de los espacios asociados a las agregaciones de funciones.

- Análisis de las interrelaciones entre agregaciones funcionales y apreciación de sus

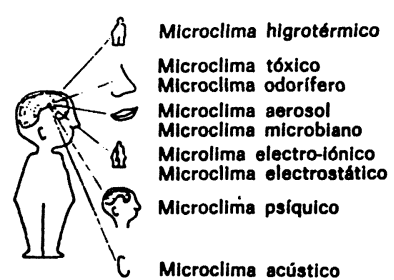

Figura 2. Tipos de microclima (adaptado de una figura por Ryd y Wyon).

\begin{tabular}{|c|c|c|}
\hline \multicolumn{2}{|c|}{ AGENTES } & MICROCLIMA \\
\hline MATERIALES & $\begin{array}{l}\text { Sollidos tóxicos } \\
\text { Aorosoles toxicos } \\
\text { Micro-organismos } \\
\text { Liquidos toxicos } \\
\text { Aorosoles liquidos } \\
\text { Gases tóxicos } \\
\text { Olores } \\
\text { Vapores de agua }\end{array}$ & $\begin{array}{l}\text { Aerosol } \\
\text { Microbiano } \\
\text { Toxico } \\
\text { Odoriforo }\end{array}$ \\
\hline ENERGETICOS & 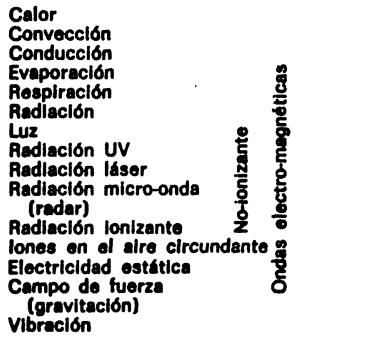 & $\begin{array}{l}\text { Eloctro-1ónico } \\
\text { Eloctrostatico } \\
\text { Acústico }\end{array}$ \\
\hline PSIOUICOS & $\begin{array}{l}\text { Espaclosided dol amblente } \\
\text { Color del amblente } \\
\text { Diaturblo mochinlco creado } \\
\text { por un movimiento de alre inadecuado } \\
\text { Roleclones humanas }\end{array}$ & Palquico \\
\hline
\end{tabular}

implicaciones en la organización espacial de las células del habitat.

Para el estudio de los ambientes interior y exterior, así como de los entornos de vida, trabajo y habitación, es preciso también analizar los modernos fundamentos de la Ecología y sus categorías.

Posiblemente sea Checoslovaquia uno de los países europeos donde se haya investigado con más seriedad científica la teoría de los entornos y de los ambientes espaciales aplicados en la edificación.

Los agentes y sus complejos, actuando sobre el usuario, producen diferentes esfuerzos que provocan un reflejo con los síntomas contingentes sobre el organismo vivo, y se puede clasificar el microclima según los tipos de esfuerzo especificados en el cuadro. (Fig. 2). El ambiente físico puede considerarse como el entorno con el cual el organismo vivo interacciona. El organismo, por su existencia y su actividad, afecta al ambiente $y$, a su vez, el ambiente afecta al organismo.

Un concepto interesante es el de ecosistema, empleado por primera vez por el botánico inglés Tansley, y que estudia el entorno de las plantas referido al clima, al suelo y a sus organismos vivos. El ecosistema complejo comprende tres grandes variables: los que pertenecen propiamente 
al ambiente (temperatura, humedad, agresión gravedad, movimiento y calidad del aire, etc.), con que se refieren al organismo vivo (edad, sexo, peso, presión arterial, metabolismo, características psicológicas, etc.); y aquellos que median entre los dos citados (alimentación, vestimenta, valores y actividades sociales, etc.).

Sería desproporcionando prolongar este artículo, ya que su objeto es simplemente dar una referencia de los temas tratados en la importante Conferencia Internacional de la EUROBUILD, que constituyen una panorámica de los problemas importantes actuales y futuros de la construcción.

Solamente se desea añadir que, así como los años sesenta se caracterizaron por la preocupación de conseguir una racionalización y una industrialización del sector a base de elementos prefabricados, y en la segunda mitad de los setenta apareció la gran preocupación por el ahorro de energía en el diseño y en la fabricación de elementos y en la concepción de los sistemas, el desafío actual con el que se enfrenta todo aquel que interviene en el proceso de la construcción, es el que proviene de la rápida invasión de la Informática y de la Electrónica.

Estemos, pues, al día e incorporemos los más modernos conocimientos a nuestras investigaciones, para poder entregar tal vez nuestro grano de arena para la creación y realización de un habitat que satisfaga de una manera óptima a las exigencias del hombre del mañana.

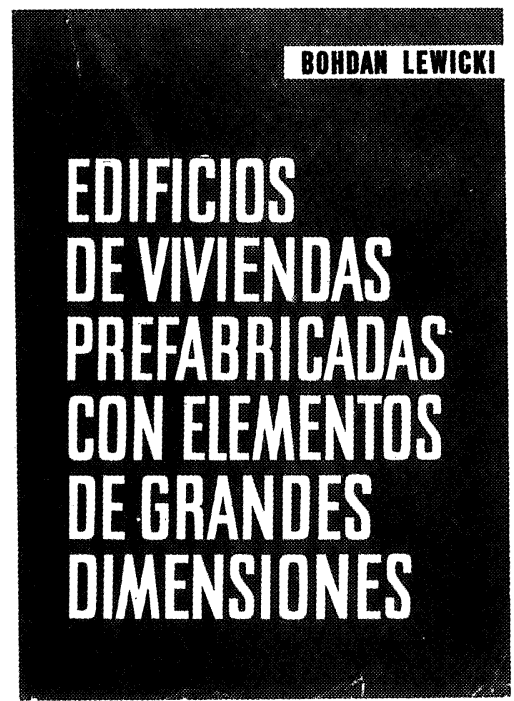

Bohdan Lewicki

Este libro trata de los problemas relativos a la construcción de los edificios de viviendas o publicos realizados con elementos prefabricados de grandes dimensiones. Se han estudiado los problemas de arriostramiento asi como los que plantea la resistencia de los examinado las cuestiones de orden higrotérmico, acústico y de resistencia al fuego; también se ha profundizado en el estudio de la estanquidad de los muros exteriores y de las juntas.

La obra incluye numerosas ilustraciones que dan detalles de diversas soluciones, asi como ejemplos de cálculo, tablas de valores numéricos, diagramas y ábacos.

Un volumen encuadernado en tela, de $24 \times 17 \mathrm{~cm}$, compuesto de 616 págs.

Precios: 2.500 ptas.; \$USA 36.00 .

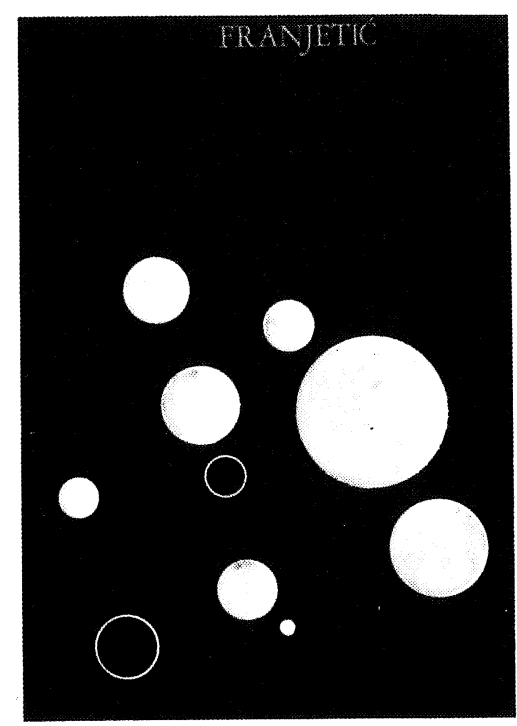

Zorislav Franjetić

En la obra de Franjetic se expone de una forma minuciosa, ordenada y sistemática todo un cuerpo de doctrina que reúne el conocimiento actual sobre el endurecimiento rápido del hormigón. Parte el autor de los principios básicos y llega a las últimas consecuencias y realidades técnicas y economicas.

Es una obra de consulta, tanto para el investigador sobre la materia, como para el proyectista y el realizador y montador de plantas e instalaciones y equipos de curado y endurecimiento rápido.

Un volumen encuadernado en cartóné, de $17 \times 24,5 \mathrm{~cm}$, compuesto de 385 págs. 110 figuras y 10 tablas.

Precios: 2.500 ptas.; \$USA 36.00

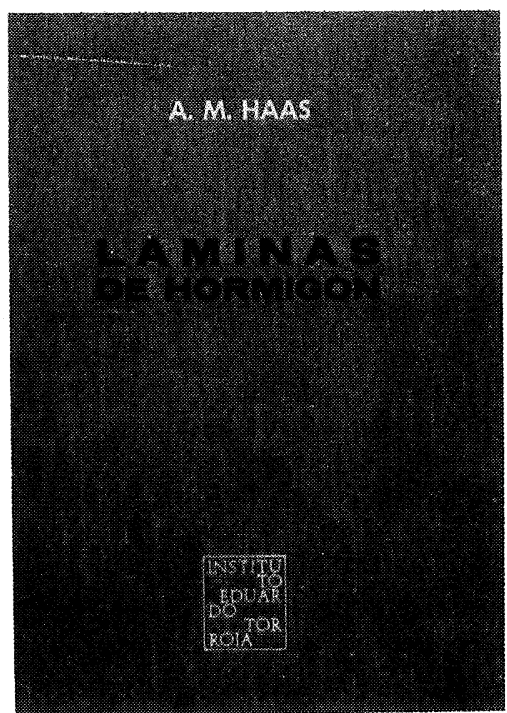

A. M. Haas

Al escribir este libro el autor intento poner a disposición de los estudiantes y de los ingenieros unos conocimientos prácticos, adecuados para servir de guia en el diseño y construcción de láminas delgadas de hormigón.

El autor está convencido de que el éxito en el diseño de una lámina exige, por parte del proyectista, un examen de las tres fases por las que pasa la materialización de la lámina el diseño, el análisis estructural y la construcción de la estructura.

Un volumen encuadernado en tela, de $17 \times 24,5 \mathrm{~cm}$, compuesto de 420 págs., 141 figuras, 22 fotografías y 6 tablas.

Precios: 2.500 ptas.; \$USA 36.00 